\title{
3 Research Square \\ The Demographic Characteristics Affecting Fear of Falling Avoidance Behaviors in Older Adults
}

\section{Sohail Jamaati}

The University of Social Welfare and rehabilitation Sciences

\section{Enayatollah Bakhshi}

The University of Social Welfare and rehabilitation Sciences

Hojjat A. Haghgoo ( $\square$ haghgooh@gmail.com )

The University of Social Welfare and rehabilitation Sciences

\section{Research Article}

Keywords: Fear of Falling Avoidance Behavior Questionnaire, Fear of falling, Avoidance Behavior, Elder

Posted Date: August 24th, 2021

DOI: https://doi.org/10.21203/rs.3.rs-789525/v1

License: (c) (1) This work is licensed under a Creative Commons Attribution 4.0 International License. Read Full License 


\section{Abstract}

Purpose: Not only falling but also fear of falling deeply affects people's social participation and quality of life. We aimed to investigate the relationship between demographic characteristics and the fear of falling and consequently the life habits and satisfaction in elderly people.

Methods: In a cross-sectional study, a total of 203 people aged over 60 years were recruited in Tehran, and assessed using the Fear of Falling Avoidance Behaviors Questionnaire, Falls Efficacy Scale (FES-1), and Life Habits tests (Life-H). Data were used to measure the prevalence of fear of falling and its correlation with life habits.

\section{Results:}

Correlation coefficient of total score of the questionnaire was directly correlated with Fall efficacy scale ( $p$ $<0.001, r=0.94)$, and inversely correlated with Activity $(p=0.004, r=-0.51)$, and Satisfaction levels $(p=0$ $(003, r=-0.53)$ of Life-Habits questionnaire.

There were significant correlations between age and FFABQ scores $(r=28 \%, P<0.001)$, educational level $(p<0.000)$, and also significant relation between gender and fear of falling $(p=0.006)$. The chi-square test showed a significant relationship between the history of falling with fear of falling $(p<0.001)$.

Conclusion: fear of falling severely affects life habits and decreases Iranian older people's social activity and participation. It is strongly affected by demographic characteristics such as gender, age, and education.

Trial registration number: IR.USWR.REC.1398.093

Date of registration: $4^{\text {th }}$ August 2019

\section{Introduction}

Demographic developments are now in a wave of shifting toward the elderly [1]. The increase in the number of elderly people is accompanied by an increase in the number of diseases and problems that this group of the population will face. Falling is the most common problem in the elderly, which can lead to fractures, disabilities, and even death. The World Health Organization (WHO) has identified falling as the third leading cause of chronic disability in the world [2]. Also, according to the Centers for Disease Control and Prevention (CDC), the total cost of the falling in the United States in 2013 was $\$ 34$ billion, and more than 700,000 patients are hospitalized each year due to falls [3]. The prevalence of falling will increase with age so that the prevalence in the elderly aged 65 and over is 28 to 35 percent, and in the elderly aged 75 years and older is 32 to 42 percent $[4,5]$. Falling is usually caused by physiological disorders such as balance problems, muscle weakness, and decreased reaction time [6]. Falling will have a devastating effect on the physical, psychological, social, and economic well-being of the elderly [4]. The psychological consequences of falling will include fear of falling, loss of self-esteem and self-confidence, 
as well as avoidance of activity [7]. Accidents caused by falls and their consequences are major obstacles in the daily activities of the elderly, and reduce self-esteem, reduce the social participation of the elderly and reduce the quality of life of these people $[8,9]$.

Aside from falling and its consequences, the fear of falling is one of the problems that interfere with participation and activity in the elderly. Researchers describe the fear of falling as a chronic concern about falling, which ultimately limits performance in daily activities [7]. Some researchers also point to the fear of falling as a lack of self-confidence in balance skills. They believe that the fear of falling can be more worrying than falling [10]. On average, 25 to 55 percent of the elderly report a fear of falling, of which 20 to 55 percent experience activity restriction due to fear of falling [11]. Research shows that $50 \%$ of the elderly population will be afraid of falling even before the first experience of falling [12]. While $25 \%$ of people are injured after a fall, $35 \%$ will be afraid of falling. Also, $45 \%$ of people are unable to get up and recover after a fall [13]. However, falling is not a prerequisite to fear of falling [14, 15]. Howland et al 1993 reported that 20 percent of people who have not recently experienced a fall are still more or less afraid of falling. Thus, in elderly people, with and without a history of falling, the fear of falling may lead to inactivity, social isolation, and consequently reduced performance and decreased quality of life $[15,16]$.

The main goal of the rehabilitation process is to help people return to their maximum activity and social participation. Therefore, the important goal of treatment in rehabilitation programs is decreasing dependency, and increasing social life and social participation $[17,18]$. Knowing demographic factors affecting fear of falling behaviors can help in predicting and preventing the behavior in the targeted population. In the present work, we aimed to investigate the demographic characteristics which affect the fear of falling behaviors, and the relation between these factors and life habits in the Iranian elderly.

\section{Materials And Method}

This study was approved by the ethical committee of the University of Social welfare and Rehabilitation Sciences, Tehran, Iran (IR.USWR.REC.1398.093) as a descriptive nonexperimental methodological study and was conducted in accordance with the principles of the Declaration of Helsinki. All participants were provided with the information sheet and ensured that their participation in the research is voluntary and they were able to withdraw from the study in every stage of the data collection process. Following their consent, data were collected at the participant's convenient time and day. All the elderly people who provided consent were included in the study.

\section{Participants}

A total of 203 elderly people (over 60 years), with and without a history of falling were recruited by availability [28] from Karaj and Tehran elderly people considering inclusion 
criteria. Inclusion criteria were: 1 . Willingness to take part in the study, 2. age 60 or older, 3. absence of a psychological disorder, and 4. having a normal cognitive status i.e. the MMSE score above 22 in literate people and above 17 in illiterate people.

\section{Data collection:}

Data collection was conducted in a quiet room with proper lighting and ventilation or in a calm and relaxed place. There were five tools for collecting data including:

1. MMSE with 6 subscales for orientation, registration, attention, calculation, and language and praxis tests was used to estimate the participant's cognitive abilities. In this study, literate participants with scores 22 and higher, and illiterate participants with scores 17 and higher were included in the study.

2. A questionnaire within which data on demographic data including age, gender, marital status, and history of psychological disorders were collected.

3. The FFABQ was fulfilled either by interviewing (for illiterate or disabled people) or by submitting to the subjects (literate and capable people) and they individually answered and completed the FFABQ Questionnaire.

The FFABQ: The "Fear of Falling Avoidance Behavior Questionnaire" (FFABQ) measures the avoidance behavior that restricts activity and participation concerning the fear of falling. It was developed by Lethem et al 1983 [19]and Troup et al 1987 [20]based on the "model of avoidant fear of exaggerated pain perception". This model explains the psychological and mental components of avoiding activities. In this model, the individual learns through operational conditioning that when he is in a condition of fear or stressful stimulus, he avoids that condition or stimulus [21]. The basis of the FFABQ hypothesis is that people who are afraid of falling because of a history of falling or secondary to the negative consequences of falling and reverberate thoughts related to the fear of falling in their minds, avoid activities that put them at risk. Therefore, the importance of the FFABQ is in identifying the activities that the person avoids due to fear[16]. This questionnaire includes 14 items to measure the "result" of fear of falling (not the fear of falling) based on the International Classification of Function, Disability, and Health (ICF) at the activity and participation levels. Rating is in the range of 0 to 56 , and a higher score indicates more avoidance behaviors. Each item scores from 0 to 4 in such a way that $0=$ completely 
disagree, 1 =disagree, 2 =neutral, 3 = agree, and $4=$ completely agree. The cut-off point of the score for the avoidance behavior of fear of falling is not reported. However, the score of 20 is determined as the cutting point[22]. This means that the people with scores less than 20 do not show avoiding behavior while the score of 20 or higher indicates avoiding behavior. When the score is less than 20, the average response in 14 items is in the range of "disagree" and "completely disagree". Theoretically, reporting a score lower than the cutoff point indicates a mild level of avoidance of fear of falling, and higher scores above the cut-off point indicate a moderate to severe level of avoidant behavior \{Landers, 2017 \#88\}. 4. Fall-Efficiency Scale Questionnaire International Form (FES-I): This questionnaire consisted of 16 items. The items in this questionnaire have 4 options ranging from "I'm not worried at all" to "I'm completely worried". The total score of the test is ranging from 16 to 64. A higher score means more fear of falling or lower self-efficacy [23].

5• - Life Habits Questionnaire (Life-H): this test has two general categories of daily activities and social roles, each one consisted of 6 subscales. Daily activities include: nutrition, health, personal care, communication, housework and mobility, and social roles include: responsibilities, interpersonal relationships, social life, education, employment, and recreation. This questionnaire has 77 items [24, 25].

\section{Statistics:}

The normality of data was examined by the Shapiro-Wilk test. Spearman Correlation coefficient was used to investigate the relation between the two questionnaires, fear of falling, and demographic characters such as age, gender, education, and falling history. $\mathrm{P}<0.05$ was considered significant. All statistical analyzes were performed using SPSS software version 24 .

\section{Results}

Demographic characteristics of 203 participants in the study with the age range of 60 to 93 years (mean age $=73.3 \pm 7.03$ years) are shown in Table 1 .

\section{Table 1. Demographic characteristics of the participants}




\begin{tabular}{|c|c|c|c|}
\hline Variable & level & Number & $(\%)$ \\
\hline \multirow[t]{2}{*}{ gender } & male & 105 & $(51.7 \%)$ \\
\hline & female & 98 & $(48.3 \%)$ \\
\hline \multirow[t]{3}{*}{ Cognitive Function(MMSE } & $25-30$ & 85 & $(41.9 \%)$ \\
\hline & 22-25 for literates & 118 & $(58.1 \%)$ \\
\hline & $>17$ for illiterates & & \\
\hline \multirow[t]{4}{*}{ Education status } & Illiterate & 96 & $(47.3 \%)$ \\
\hline & <Diploma & 55 & $(27.1 \%)$ \\
\hline & Diploma & 32 & $(15.8 \%)$ \\
\hline & University Degree & 20 & $(9.9 \%)$ \\
\hline \multirow[t]{6}{*}{ Special Diseases } & Blood pressure or Diabetes & 50 & $(24.6 \%)$ \\
\hline & Lower extremities' or Spinal Column Arthritis & 74 & $(36.5 \%)$ \\
\hline & Low back Pain or Dick Herniation & 14 & $(6.9 \%)$ \\
\hline & Fracture in Lower extremities or trunk & 9 & $(4.4 \%)$ \\
\hline & Tow of above conditions & 49 & $(24.1 \%)$ \\
\hline & None of them & $7(3.4 \%)$ & \\
\hline \multirow[t]{5}{*}{ Using assistive aids } & No use & 102 & $(50.2 \%)$ \\
\hline & Stick & 58 & $(28.6 \%)$ \\
\hline & Walker & 21 & $(10.3 \%)$ \\
\hline & Eye glass & 14 & $(6.9 \%)$ \\
\hline & Using two or more & 8 & $(3.9 \%)$ \\
\hline \multirow[t]{4}{*}{ Rehabilitation Services } & No service & 100 & $(49.3 \%)$ \\
\hline & Occupational Therapy & 32 & $(15.8 \%)$ \\
\hline & Physiotherapy & 11 & $(5.4 \%)$ \\
\hline & Occupational Therapy and Physiotherapy & 60 & $(29.6 \%)$ \\
\hline \multirow[t]{2}{*}{ Falling History in the past year } & Yes & 142 & $(70 \%)$ \\
\hline & No & 61 & $(30 \%)$ \\
\hline \multirow[t]{2}{*}{ Living in nursing home } & Yes & 132 & $(65 \%)$ \\
\hline & No & 71 & (35\%) \\
\hline
\end{tabular}


The results of the Shapiro-Wilk test showed that data are not distributed normally $(P=0.01 \& P=0.04$, $\mathrm{P}<0.05)$, therefore the Spearman Correlation Coefficient was used to investigate the relation between the two questionnaires. Spearman's correlation coefficient between the FES-I and FFABQ showed a significant correlation between the two questionnaires $(p<0.001)$.

Table 2: The correlations between demographic characteristics and fear of falling

\begin{tabular}{lllll} 
& History of falling & age & Gender & Education level \\
\hline Fear of falling & $\mathrm{P}<0.000$ & $\mathrm{R}=0.282 \mathrm{P}, 0.000$ & $\mathrm{~F}=13.202$ & $\mathrm{P}<0.000$ \\
& & & $\mathrm{P}<0.006$ & \\
\end{tabular}

The Chi-square test showed a significant relationship between the history of falling with fear of falling $(p<0.001)$. Among the people with a history of falling, $87 \%$ showed fear of falling avoidance behaviors, while only $31 \%$ of participants without a history of falling, showed such avoidance behaviors.

There was a significant correlation between age and FFABQ scores $(r=28 \%, P<0.001)$.

Further, Chi-test showed that there is a significant relationship between gender and fear of falling $(p=0.006)$. While $62 \%$ of male participants showed fear of falling, the percentage of females with fear of falling was $80 \%$. Additionally, the level of education showed a significant correlation with fear of falling behaviors. The higher the educational level, the lower score on the FFABQ test.

Table 3: The relationship between the score of the Life Habits Questionnaire in the two sections of activity and satisfaction levels with the FFABQ

\begin{tabular}{llll} 
Questionnaires & & Pearson Correlation Coefficient & P. Value \\
\hline FFABQ & Activity Level & -0.51 & 0.004 \\
\hline & Satisfaction Level & -0.53 & 0.003 \\
\hline Activity Level & Satisfaction Level & 0.59 & 0.001
\end{tabular}

The results showed a significant correlation between the Life's Habits Questionnaire and the FFABQ questionnaire $(R=-60 \%, p<0.001)$. The results showed significant relationships between the score of the Life Habits Questionnaire in the two sections of activity and satisfaction levels with the FFABQ questionnaire score was obtained using Pearson correlation coefficient (Table 3).

The results showed significant negative correlations between activity and satisfaction levels and the FFABQ questionnaire score. Furthermore, a significant correlation between activity level and satisfaction level was observed in the elderly. Another measure was made to verify the detailed correlation between the FFABQ test and life habit subscales (Table 4).

Table 4: the detailed correlation between the FFABQ test and life habit subscales 


\begin{tabular}{llll} 
Questionnaires & Life-H & Pearson Correlation Coefficient & P. Value \\
\hline FFABQ & Nutrition & -0.414 & $\mathbf{0 . 0 2 3}$ \\
\hline Health & -0.493 & $\mathbf{0 . 0 0 6}$ \\
\hline Personal care & -0.482 & $\mathbf{0 . 0 0 7}$ \\
\hline Communication & -0.225 & 0.231 \\
\hline House work & -0.390 & $\mathbf{0 . 0 3 3}$ \\
\hline Mobility & -0.400 & $\mathbf{0 . 0 2 9}$ \\
\hline Responsibility & -0.113 & 0.551 \\
\hline Interpersonal relationship & 0.035 & 0.856 \\
\hline Social life & -0.300 & 0.107 \\
\hline Education & -0.254 & 0.176 \\
\hline Employment & -0.324 & 0.081 \\
\hline Recreation & -0.539 & $\mathbf{0 . 0 0 2}$ \\
\hline Total & -0.455 & $\mathbf{0 . 0 1 2}$
\end{tabular}

As shown in table 4. Fear of falling behaviors is correlated significantly with different life habits such as nutrition, health, personal care, housework, mobility, and recreation.

\section{Discussion}

The purpose of this study was to investigate the relationship between demographic characteristics on the fear of falling behaviors and the effects of these avoidance behaviors on life habits and social participation.

According to the results, the level of activity of the elderly has a significant correlation with the rate of avoidance behavior of fear of falling. As the elderly's avoidance behaviors increase, their level of activity in the environment will decrease, and vice versa. This finding is consistent with the findings of Joshi et al. 2015[24] who reported that the elderly avoid certain ADLs or IADL activities because of fear of falling. These activities mainly involve walking in crowded places, lifting and carrying objects, doing housework (like cleaning, washing clothes), recreational activities, and leisure. These people try to avoid activities that may put them at risk. Therefore, they severely restrict parties, entertainment, and social activities [24]. In another study by Ulrich et al. in the elderly after discharge from rehabilitation, it was reported that factors related to fear of falling are effective in mobility in the living environment [26].

Another finding in this study showed a significant relationship between the avoidance behavior because of fear of falling and the level of elderly satisfaction with ADL and IADL. An increase in the elderly's 
avoidant behaviors due to the fear of falling results in decreases in his level of satisfaction with daily activities, and vice versa. Joshi [27] has reported that avoidance behavior due to fear of falls affects the quality of life in the elderly by restricting social participation [27]. In the study of Lachman et al., It was also reported that in extreme forms of fear of falling, this fear significantly reduces daily activities due to disabling anxiety during activities [10]. This disabling anxiety definitely reduces the level of satisfaction with activities in the elderly.

The results showed that fear of falling behavior can affect the nutrition, health, and personal care in the elderly and restricts their mobility and housework duties. In addition, these avoiding behaviors affect older people's tendency to participate in recreational activities. Considering the importance of social integration and social participation for a productive and dynamic life, these restrictions play key roles in isolated older people.

Furthermore, present results showed that fear of falling behaviors significantly correlates with older ages and lower education. These behaviors are more prevalent in females than males. Considering these results, it is necessary to plan especially rehabilitation programs and pay attention carefully to females, the people with lower levels of education, the people with a history of falling in the older people

Some limitations may restrict the generalizing of the results. Most people in this study were in nursing homes. Living in nursing homes is effective in increasing the level of avoidance behaviors in people. Conclusion:

\section{Conclusion}

Not only falling but fear of falling lead to avoidance behaviors. These avoidance behaviors affect life habits including social participation and satisfaction. These avoidance behaviors can be affected by demographic characteristics such as age, gender, and education in addition to a history of falling. Especial attention should be paid to females with lower education and higher age.

\section{Declarations}

\section{Funding: The University of Social Welfare and Rehabilitation Sciences, Tehran, Iran, which approved the ethics, and supports the research by providing the clinical setting and data-gathering instruments.}

Compliance with Ethical Standards: : This study was approved by the ethical committee of the University of Social welfare and Rehabilitation Sciences, Tehran, Iran (IR.USWR.REC.1398.093). All participants were provided with the information sheet and ensured that their participation in the research is voluntary and they were able to withdraw from the study in every stage of the data collection process. Following their consent, data were collected at the participant's convenient time and day.

Informed consent: attached as appendix. 
Competing interest: 'The Author(s) confirms that there is no conflict of interest, no Competing interest'. Ethical approval: This clinical trial was approved by the Ethic Committee of the University of Social Welfare and Rehabilitation Sciences Iran (IR.USWR.REC.1398.093)

Consent for publication: This manuscript that represents original work is not under review or consideration by another journal and has not been published by another journal

Availability of data and material: Data are available on: https://data.mendeley.com/drafts/7c53s35t44 and no administrative permissions are required to access the raw data from the above site.

Authors' contributions: all the authors were involved in the research. S.J. in conducting research and data gathering, E.B. in analyzing data and reporting the results, H.H. in designing the research, interpreting data, writing the manuscript and submitting. all authors have read and approved the manuscript".

\section{Acknowledgments:}

The authors would like to thank the officials of Kahrizak Elderly Center in Karaj, Iran for their cooperation. We would also like to express our sincere gratitude to all the elderly people who participated in this research with patience.

\section{References}

1. Organization, W.H., World report on ageing and health. 2015: World Health Organization.

2. Van Schoor, N.M., et al., Different cognitive functions in relation to falls among older persons: immediate memory as an independent risk factor for falls. 2002. 55(9): p. 855-862.

3. Ogden, C.L., et al., Centers for Disease Control and Prevention 2000 growth charts for the United States: improvements to the 1977 National Center for Health Statistics version. 2002. 109(1): p. 45-60.

4. Fillit, H.M., K. Rockwood, and J.B. Young, Brocklehurst's textbook of geriatric medicine and gerontology e-book. 2016: Elsevier Health Sciences.

5. Formiga, F., et al., Factors associated with hip fracture-related falls among patients with a history of recurrent falling. 2008. 43(5): p. 941-944.

6. Carter, N.D., P. Kannus, and K.J.S.m. Khan, Exercise in the prevention of falls in older people. 2001. 31(6): p. 427-438.

7. Legters, K.J.P.t., Fear of falling. 2002. 82(3): p. 264-272.

8. Jung, D.J.A.N.R., Fear of falling in older adults: Comprehensive review. 2008. 2(4): p. 214-222.

9. Visschedijk, J., et al., Fear of falling after hip fracture: a systematic review of measurement instruments, prevalence, interventions, and related factors. 2010. 58(9): p. 1739-1748. 
10. Lachman, M.E., et al., Fear of falling and activity restriction: the survey of activities and fear of falling in the elderly (SAFE). 1998. 53(1): p. P43-P50.

11. Murphy, S.L., et al., The development of fear of falling among community-living older women: predisposing factors and subsequent fall events. 2003. 58(10): p. M943-M947.

12. Tinetti, M.E., M. Speechley, and S.F.J.N.E.j.o.m. Ginter, Risk factors for falls among elderly persons living in the community. 1988. 319(26): p. 1701-1707.

13. Baker, S. and A. Harvey, Fall injuries in the elderly-Clinics in Geriatric Medicine. 1985, Philadelphia: WB Saunders.

14. Downton, J. and K.J.I.J.o.G.P. Andrews, Postural disturbance and psychological symptoms amongst elderly people living at home. 1990. 5(2): p. 93-98.

15. Howland, J., et al., Fear of falling among the community-dwelling elderly. 1993. 5(2): p. 229-243.

16. Landers, M.R., et al., Development of a scale to assess avoidance behavior due to a fear of falling: the Fear of Falling Avoidance Behavior Questionnaire. 2011. 91(8): p. 1253-1265.

17. Hadjistavropoulos, H.D., et al., Development and evaluation of a Continuity of Care Checklist for improving orthopaedic patient discharge from hospital. 2009. 13(4): p. 183-193.

18. Liyun, X.J.C.M.H., Effect of systematic nursing intervention on elderly femoral neck fracture patients. 2010. 18(6): p. 97-100.

19. Lethem, J., et al., Outline of a fear-avoidance model of exaggerated pain perception-I. 1983. 21(4): p. 401-408.

20. Troup, J., et al., The perception of back pain and the role of psychophysical tests of lifting capacity. 1987 Volvo Award in Clinical Sciences. 1987. 12(7): p. 645-657.

21. Slade, P., et al., The fear-avoidance model of exaggerated pain perception-II: Preliminary studies of coping strategies for pain. 1983. 21(4): p. 409-416.

22. Landers, M.R., et al., A cross-sectional analysis of the characteristics of individuals with Parkinson disease who avoid activities and participation due to fear of falling. 2017. 41(1): p. 31-42.

23. Yardley, L., et al., Development and initial validation of the Falls Efficacy Scale-International (FES-I). 2005. 34(6): p. 614-619.

24. Desrosiers, J., et al., Participation in daily activities and social roles of older adults with visual impairment. 2009. 31(15): p. 1227-1234. 
25. Viscogliosi, C., et al., Participation after a stroke: Changes over time as a function of cognitive deficits. 2011. 52(3): p. 336-343.

26. Ullrich, P., et al., Life-space mobility in older persons with cognitive impairment after discharge from geriatric rehabilitation. 2019. 81: p. 192-200.

27. Joshi, S. and S.R.J.W.w.i.c. Joshi, Effect of Fear of Falling on Quality of Life in Geriatric Population. 2015. 9(4): p. 173. 